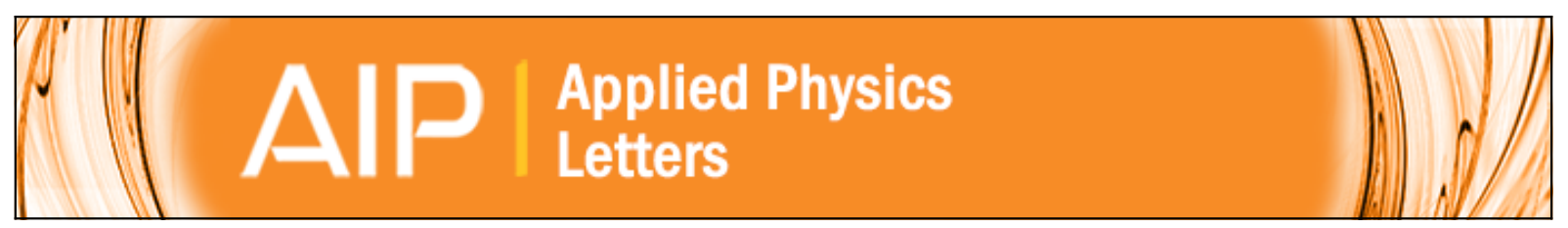

\title{
Simultaneous enhancement of electron overflow reduction and hole injection promotion by tailoring the last quantum barrier in InGaN/GaN light-emitting diodes
}

Zabu Kyaw, Zi-Hui Zhang, Wei Liu, Swee Tiam Tan, Zhen Gang Ju, Xue Liang Zhang, Yun Ji, Namig Hasanov, Binbin Zhu, Shunpeng Lu, Yiping Zhang, Jing Hua Teng, Sun Xiao Wei, and Hilmi Volkan Demir

Citation: Applied Physics Letters 104, 161113 (2014); doi: 10.1063/1.4873395

View online: http://dx.doi.org/10.1063/1.4873395

View Table of Contents: http://scitation.aip.org/content/aip/journal/apl/104/16?ver=pdfcov

Published by the AIP Publishing

\section{Articles you may be interested in}

Improved carrier injection and efficiency droop in InGaN/GaN light-emitting diodes with step-stage multiplequantum-well structure and hole-blocking barriers

Appl. Phys. Lett. 102, 241108 (2013); 10.1063/1.4811735

Improved hole distribution in InGaN/GaN light-emitting diodes with graded thickness quantum barriers

Appl. Phys. Lett. 102, 243504 (2013); 10.1063/1.4811698

Droop improvement in blue InGaN/GaN multiple quantum well light-emitting diodes with indium graded last barrier

Appl. Phys. Lett. 99, 233501 (2011); 10.1063/1.3665252

Hole transport improvement in InGaN/GaN light-emitting diodes by graded-composition multiple quantum barriers

Appl. Phys. Lett. 99, 171106 (2011); 10.1063/1.3655903

Hole injection and efficiency droop improvement in InGaN/GaN light-emitting diodes by band-engineered electron blocking layer

Appl. Phys. Lett. 97, 261103 (2010); 10.1063/1.3531753

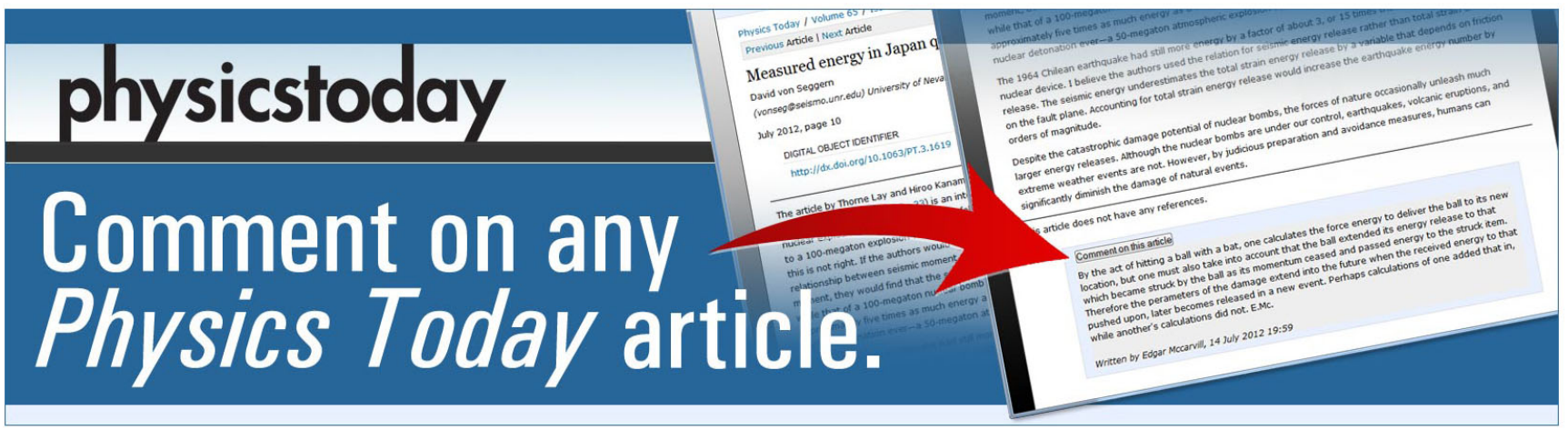




\title{
Simultaneous enhancement of electron overflow reduction and hole injection promotion by tailoring the last quantum barrier in InGaN/GaN light-emitting diodes
}

\author{
Zabu Kyaw, ${ }^{1}$ Zi-Hui Zhang, ${ }^{1}$ Wei Liu, ${ }^{1}$ Swee Tiam Tan, ${ }^{1}$ Zhen Gang Ju, ${ }^{1}$ Xue Liang Zhang, ${ }^{1}$ \\ Yun Ji, ${ }^{1}$ Namig Hasanov, ${ }^{1}$ Binbin Zhu, ${ }^{1}$ Shunpeng Lu, ${ }^{1}$ Yiping Zhang, ${ }^{1}$ Jing Hua Teng, ${ }^{2}$ \\ Sun Xiao Wei, ${ }^{1}$ and Hilmi Volkan Demir ${ }^{1,3,4, a)}$ \\ ${ }^{1}$ LUMINOUS! Centre of Excellence for Semiconductor Lighting and Displays, School of Electrical and \\ Electronic Engineering, Nanyang Technological University, 50 Nanyang Avenue, Singapore 639798 \\ ${ }^{2}$ Institute of Materials Research and Engineering, Agency for Science, Technology and Research (A*STAR), \\ Singapore 117602 \\ ${ }^{3}$ School of Physics and Mathematical Sciences, Nanyang Technological University, 21 Nanyang Link, \\ Singapore 637371 \\ ${ }^{4}$ Department of Electrical and Electronics, Department of Physics, and UNAM-Institute of Material Science \\ and Nanotechnology, Bilkent University, TR-06800 Ankara, Turkey
}

(Received 12 January 2014; accepted 11 April 2014; published online 24 April 2014)

\begin{abstract}
A three-step graded undoped-InGaN layers embedded between the GaN last quantum barrier layer and the p-AlGaN electron blocking layer was proposed and its effect on the performance of InGaN/GaN light-emitting diodes was investigated both experimentally and theoretically. In the proposed structure, the electron leakage is found to be effectively reduced, while the hole injection efficiency is simultaneously increased significantly, hence enabling a greatly enhanced radiative recombination rate within the active region. As a result, improvements of $12.25 \%$ in the optical output power and $11.98 \%$ in the external quantum efficiency are obtained from the proposed device with the respect to the reference device. @ 2014 AIP Publishing LLC. [http://dx.doi.org/10.1063/1.4873395]
\end{abstract}

In recent years, the nitride based optoelectronic devices, including light-emitting diodes (LEDs) and laser diodes (LDs), are promising a revolution in energy-efficient lighting and displays by replacing the conventional incandescent and/or fluorescent lamps, ${ }^{1}$ and remarkable progress has been thus far achieved in the performance of these devices. To date, high brightness LEDs and long lifetime LDs have been realized and commercialized. ${ }^{2,3}$ However, there is still a large room for the electronic structure design and performance levels of III-nitride devices to be further improved. The performance of these solid-state devices can be largely limited by several loss mechanisms. For example, electron leakage out of the multiple quantum wells (MQWs) active region $^{4-6}$ and low hole injection efficiency ${ }^{7-9}$ are two of such main loss mechanisms.

A p-type high energy band gap AlGaN electron blocking layer (EBL) behind the active region is conventionally used to improve the electron confinement within the QWs and to suppress the electron leakage. Yet the p-AlGaN EBL (pEBL) also generates an unavoidable potential barrier for holes, which undesirably impedes the hole transport into the MQWs active region. An increased $\mathrm{Al}$ content in the EBL for a stronger electron confinement simultaneously makes it more difficult for holes to enter the MQWs. Thus, several InGaN/GaN LEDs with novel p-EBL designs have been proposed $^{10-12}$ to mitigate the problem. Moreover, an n-type doped EBL structure, ${ }^{13}$ an InGaN staircase electron injector, ${ }^{14}$ and polarization matched AlGaInN quantum barriers $(\mathrm{QBs})^{6}$ have also been proposed to suppress the electron leakage. Meanwhile, it has been reported that the hole

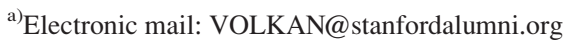

injection efficiency is improved by tailoring the last quantum barrier (LQB) in InGaN/GaN light-emitting diodes without sacrificing the electrons confinement capability. ${ }^{15-21}$ For examples, inserting a p-InGaN layer before the p-EBL, ${ }^{15-18}$ applying a $\mathrm{p}$-InGaN/GaN superlattice as the LQB,${ }^{19} \mathrm{p}$-type doping LQB, ${ }^{20}$ and thinning the $\mathrm{LQB}^{21}$ have previously been proposed. However, all of these approaches have to deal with the Mg back-diffusion to the last few QWs. These Mg dopants introduce deep nonradiative recombination levels and severely weaken the radiative recombination in QWs, particularly in the last few QWs, which actually contribute the most to the light generation among other QWs. However, these methods have their own limitations including the main one on the incapability of addressing both the factors simultaneously. Also, typically these special epitaxial layers are difficult to grow and typically adversely influence the radiative recombination rate.

As it is well known, in the conventional GaN LQB device, electron leakage is due to several reasons including carrier escape from QWs, incomplete carrier capture by QWs, and reduced barrier heights of the LQB and the p-EBL. ${ }^{6,10}$ In the [0001] oriented InGaN/GaN epi-films, the electric field is induced by the positive sheet charges at the GaN LQB/pEBL interface due to the spontaneous and piezoelectric polarization effects and hence the corresponding electric fields in the GaN LQB region and the p-EBL reduce the respective conduction barrier heights, resulting in severe electron leakage. The corresponding electric field extracts electrons from the QW, leading to a significant decrease in internal quantum efficiency. The electrons escaping from the GaN LQB region flow over the $\mathrm{p}-\mathrm{AlGaN}$ EBL to the $\mathrm{p}-\mathrm{GaN}$ due to the lower effective potential barrier height for 
electrons at the GaN LQB/p-EBL interface. It should be noted that the upward energy slope in the p-EBL actually helps prevent electron overflow, but its effect is usually overwhelmed by the lowering of the effective potential barrier height. The electron leakage leads to insufficient hole supply into the active region because the electrons flowing into the $\mathrm{p}-\mathrm{GaN}$ region recombine with holes in $\mathrm{p}-\mathrm{GaN}$ region. ${ }^{22,23}$

In this work, we proposed and demonstrated an electronic structure comprising three-step undoped-InGaN grading from low In content to high In content (designed with $\mathrm{In}_{0.015} \mathrm{Ga}_{0.985} \mathrm{~N} / \mathrm{In}_{0.052} \mathrm{Ga}_{0.948} \mathrm{~N} / \mathrm{In}_{0.09} \mathrm{Ga}_{0.91} \mathrm{~N}$ ) inserted between the GaN LQB and the p-EBL. The proposed three-step undoped-InGaN LQB simultaneously both reduces electron overflow (by suppressing the electron leakage) and promotes hole injection (by improving the hole transport). The three-step undoped-InGaN LQB architecture epi-design and structure does not add any difficulty to the growth process or cause any degradation of the radiative recombination. In the proposed structure, we systematically investigated the underlying physical mechanisms of electron overflow reduction and hole injection promotion. It has been consistently found both experimentally and theoretically that this design with the three-step graded undoped-InGaN LQB can help to suppress the electron overflow and at the same time enhance the hole injection efficiency more effectively compared to the conventional GaN LQB region. The low In content at the interface of GaN LQB will increase effective potential barrier height for electrons (by suppressing the electron leakage) and better hole transport into QWs, while high In content at the interface of $\mathrm{p}$-AlGaN EBL will decrease the effective potential barrier height for holes, promoting hole injection from hole source $\mathrm{p}$-GaN. Consequently, enhanced optical output power and external quantum efficiency (EQE) from the proposed devices have been demonstrated.

In this study, two sets of InGaN/GaN LED wafers (i.e., the reference device and the proposed device) were grown by metal-organic chemical vapor deposition (MOCVD) system. The epitaxial growth of the two LEDs was initiated from $\mathrm{c}^{+}$-sapphire substrates. Both the LEDs consisted of a $4 \mu \mathrm{m}$ thick unintentionally doped $\mathrm{GaN}(\mathrm{u}-\mathrm{GaN})$ layer grown on a 30 $\mathrm{nm}$ GaN buffer layer, followed by a $2 \mu \mathrm{m}$ thick Si-doped GaN layer $(\mathrm{n}-\mathrm{GaN})$ with the doping concentration of $5 \times 10^{18}$ $\mathrm{cm}^{-3}$. The six-period $\operatorname{In}_{0.13} \mathrm{Ga}_{0.87} \mathrm{~N} / \mathrm{GaN}$ MQWs with $3 \mathrm{~nm}$ thick well and $12 \mathrm{~nm}$ thick barrier were grown after the $\mathrm{n}-\mathrm{GaN}$ region. In composition in $\operatorname{In}_{\mathrm{x}} \mathrm{Ga}_{1-\mathrm{x}} \mathrm{N}$ was controlled by adjusting TMIn flow during InGaN growth. For the reference device, a $17 \mathrm{~nm}$ thick GaN LQB was grown following the last QW. For the proposed device, an $8 \mathrm{~nm}$ thick GaN barrier followed by three-step u-InGaN layers of $3 \mathrm{~nm}$ each with graded In content from low to high (namely, $\mathrm{In}_{0.015} \mathrm{Ga}_{0.985} \mathrm{~N} / \mathrm{In}_{0.052} \mathrm{Ga}_{0.948} \mathrm{~N} /$ $\mathrm{In}_{0.09} \mathrm{Ga}_{0.91} \mathrm{~N}$ ) were grown after the last QW. Both the reference device and the proposed device had a $25 \mathrm{~nm}$ Mg-doped $\mathrm{Al}_{0.20} \mathrm{Ga}_{0.80} \mathrm{~N}$ layer as the p-EBL, and then a $200 \mathrm{~nm}$ thick $\mathrm{Mg}$-doped $\mathrm{GaN}$ (p-GaN) region with an effective hole concentration of approximately $3 \times 10^{17} \mathrm{~cm}^{-3}$.

After the MOCVD epitaxial growth, the samples were further fabricated into chips with mesa areas of $350 \times 350$ $\mu \mathrm{m}^{2}$. Ni/Au bilayer with a thickness of $5 \mathrm{~nm} / 5 \mathrm{~nm}$ was deposited and annealed to make a transparent current spreading layer, and $\mathrm{Ti} / \mathrm{Au}(30 \mathrm{~nm} / 150 \mathrm{~nm})$ metal bilayer was then laid down for both p-contact and n-contact pads. The LED samples did not include light extraction structures. Electroluminescence (EL) spectra were measured using the LED quick tester (M2442S-9A Quatek Group) and the optical power was measured by an optical integrating sphere attached to an Ocean Optics spectrometer (QE65000), which was calibrated with a standard light source. ${ }^{24}$

The EL spectra are shown in Figs. 1(a) and 1(b) for the reference device and the proposed device, respectively. It can be seen that the EL intensity from the proposed device is stronger than that of the reference device from lower injection current of $20 \mathrm{~mA}$ to higher injection current of $150 \mathrm{~mA}$. The EQE and the optical power are depicted as a function of the injection current in Fig. 2 for both the devices. Compared with the reference device, the proposed device shows higher $\mathrm{EQE}$ and larger optical power throughout the whole current range measured. The optical output power from the proposed device is $28.13 \mathrm{~mW}$, which is about $12.25 \%$ higher than that of the reference device $(25.06 \mathrm{~mW})$ at the current level of $150 \mathrm{~mA}$. The EQE of the proposed device is about $11.98 \%$ higher than that of the reference device at $150 \mathrm{~mA}$. This observed improvement of the optical power and the EQE is well attributed to the simultaneous reduction of the electron overflow and enhancement of the hole injection efficiency enabled by the proposed three-step $\mathrm{u}$-InGaN LQB, and the details are to be discussed and demonstrated subsequently.

To reveal and understand the physical mechanisms behind the improvements observed in Figs. 1 and 2, theoretical simulations have been performed. In the theoretical simulations, APSYS simulation program has been used to solve self-consistently the Poisson's equations, continuity equations, and Schrödinger equation with proper boundary conditions. The surface charges at the interfaces were calculated using the methods developed by Fiorentini et al. ${ }^{25}$ The charge density screened by the injected carriers was also determined self-consistently at current injection levels. The parameters used in the simulations, including the Auger recombination coefficients, the Shockley-Read-Hall recombination coefficient, the energy band offset ratio, and the polarization level for devices along the c-orientation, can all be found elsewhere. ${ }^{24}$

Figs. 3(a) and 3(b) present the simulated energy band profiles for the reference device and the proposed device at $20 \mathrm{~A} / \mathrm{cm}^{2}$, respectively. The significant difference in the energy band profile for the two devices lies in the LQB region interfacing with the $\mathrm{p}$-EBL. The conduction band of the proposed device bends upwards at the interface of the GaN LQB region and the three-step u-InGaN region in contrast to the downward bending of the conduction band of the reference device. At $20 \mathrm{~A} / \mathrm{cm}^{2}$, the effective potential barrier height for electrons $\left(\Delta \varnothing_{\mathbf{e}-\mathbf{L Q B}}\right)$ is calculated to be $362 \mathrm{meV}$ for the proposed device compared to that of the reference device of $210 \mathrm{meV}$ as shown in Table I. The higher energy barrier is helpful for a better electron confinement in QWs and it is caused by the accumulation of the negative polarization charges at the interface of the GaN LQB and the three-step $\mathrm{u}$-InGaN LQB. On the other hand, the conduction band is bending downward in the GaN LQB region of the reference device due to the accumulation of the positive polarization charges at the interface of the GaN LQB and the p-EBL. 

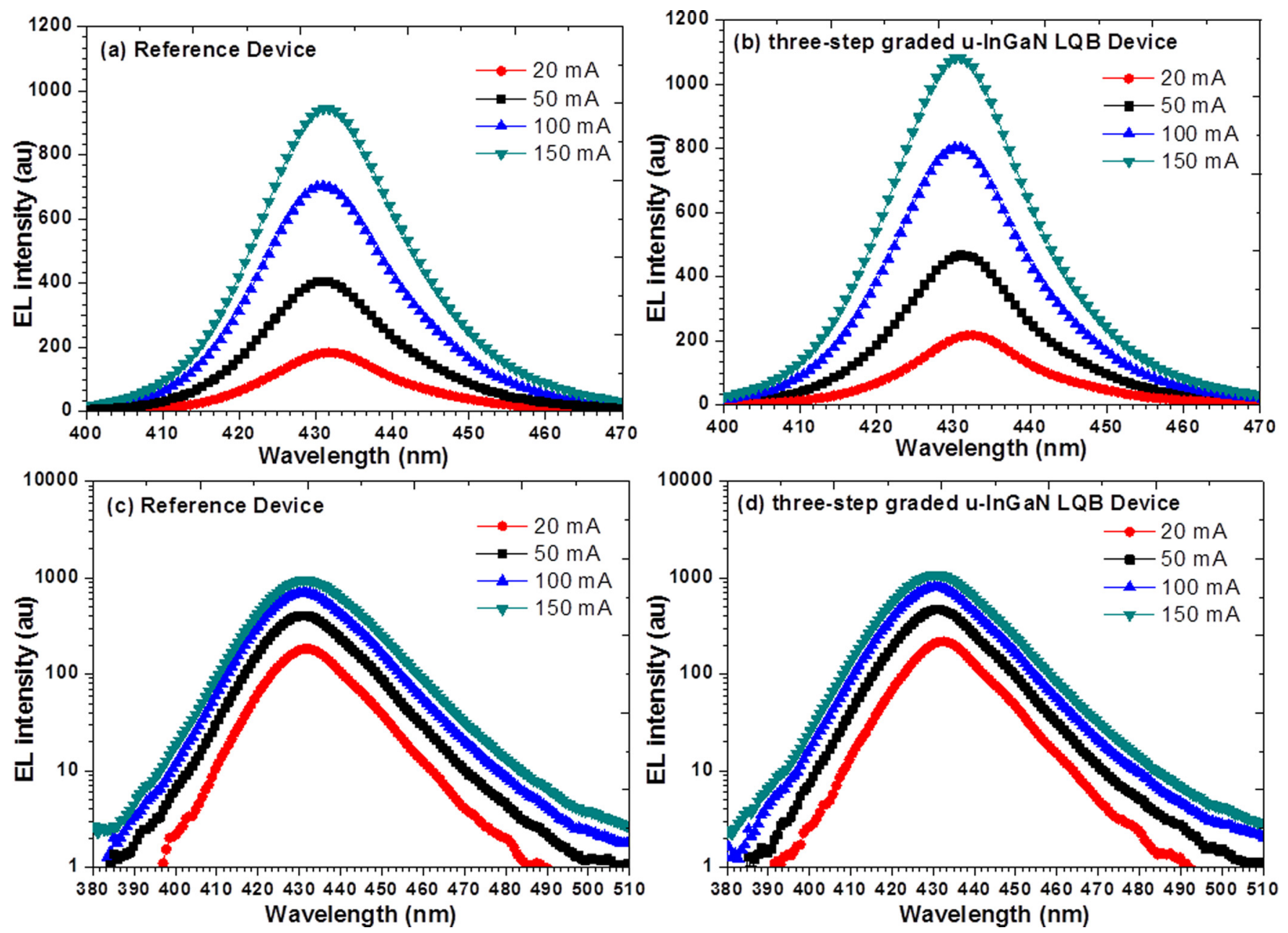

FIG. 1. EL spectra measured from (a) and (c) the reference device and (b) and (d) the proposed three-step graded u-InGaN LQB device.

The accumulation of different polarity charges at the interface of the LQB and the p-EBL for the two devices can be confirmed from the comparison of the simulated electrostatic field (E-field) in the GaN LQB region at $20 \mathrm{~A} / \mathrm{cm}^{2}$ as shown in Fig. 4(a). The positive value of the E-field represents the direction of E-field to be the same as the growth

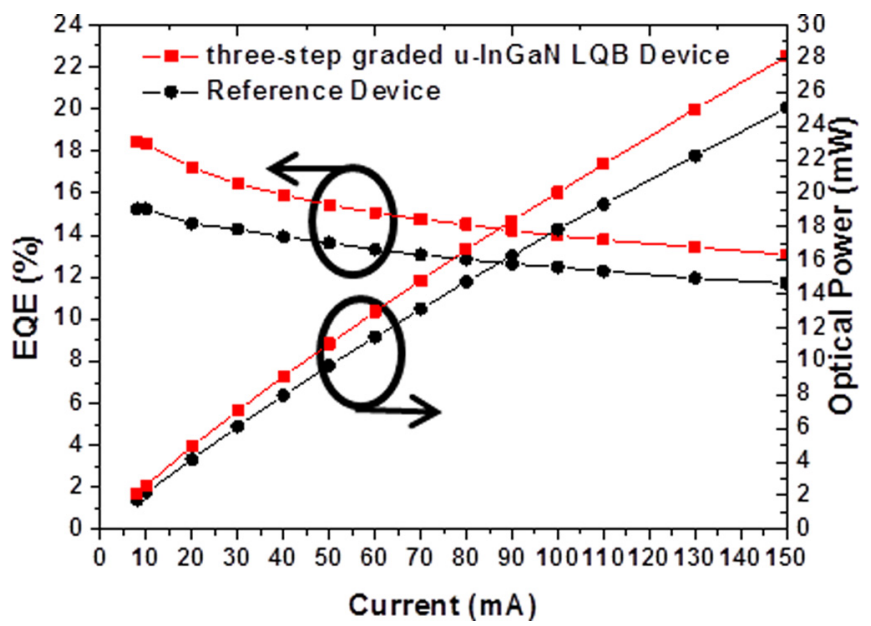

FIG. 2. Optical output power and the EQE measured as a function of the current injection for the reference device and the proposed three-step graded u-InGaN LQB device. direction $\mathrm{c}^{+}$. The positive value of the E-field was observed in the GaN LQB region of the proposed device, whereas the negative value of the E-field was observed in the GaN LQB region of the reference device. The positive value of the E-field helps in increasing the electron barrier height and improving the electron confinement in QWs of the proposed device, which is reflected in the increase of the electron concentration as shown in Fig. 4(b). This can also be further confirmed by looking at the normalized electron current as shown in Fig. 4(c). In the proposed device, a large number of electrons are consumed in the QWs regions, leaving very little leakage current in the $\mathrm{p}-\mathrm{GaN}$ region compared to that of the reference device. In addition to the improved electron confinement by the u-GaN/three-step graded u-InGaN last barrier, the increased effective potential barrier height for electrons at the three-step graded $\mathrm{u}$-InGaN LQB/p-EBL interface, which originates from the increased band offset between the $\mathrm{u}$-InGaN and the p-EBL as shown in Table I, also reduces the electron overflow. The three-step graded $\mathrm{u}$-InGaN LQB layer forms a deep potential valley in the conduction band, which in turn increases effective potential barrier height for electrons. ${ }^{16}$

The three-step graded u-InGaN LQB can also enhance the hole injection efficiency mainly due to the reduced effective potential barrier height of the p-EBL for holes. As depicted in Table I, the proposed device has presented a 

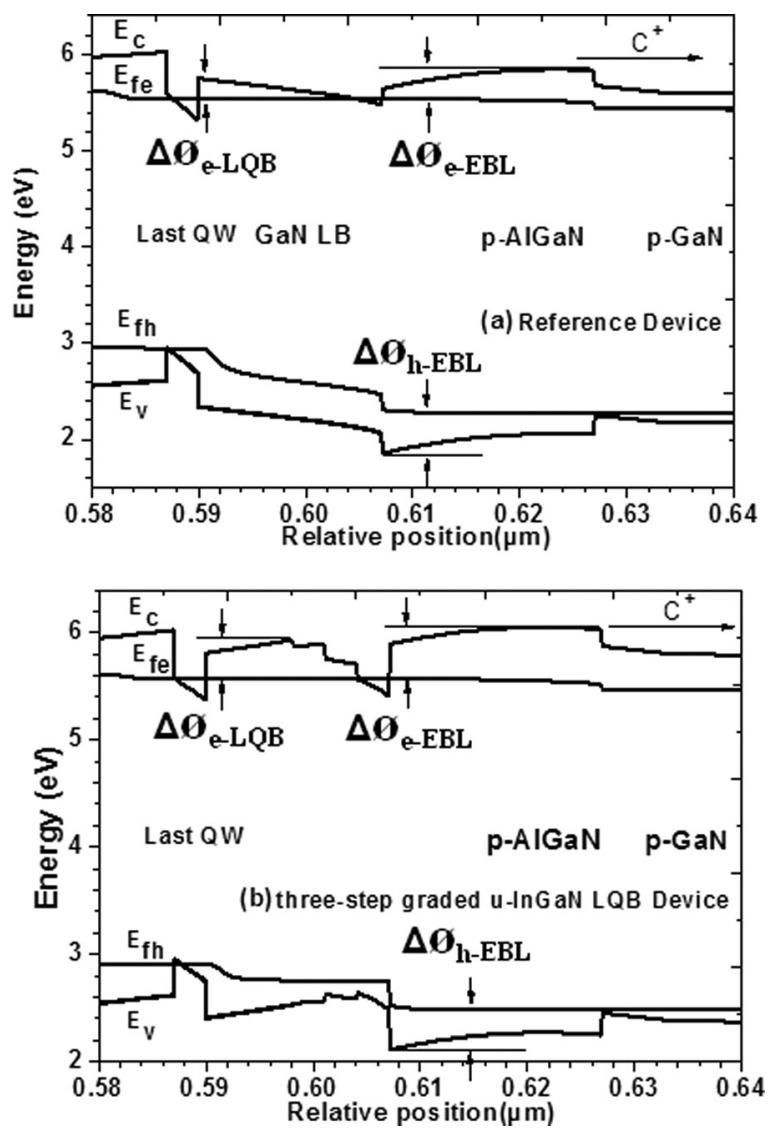

FIG. 3. Energy band diagrams calculated at $20 \mathrm{~A} / \mathrm{cm}^{2}$ for (a) the reference device and (b) the proposed three-step graded u-InGaN LQB device. Here, $\mathrm{E}_{\mathrm{c}}, \mathrm{E}_{\mathrm{v}}, \mathrm{E}_{\mathrm{fe}}$, and $\mathrm{E}_{\mathrm{fh}}$ denote the conduction band, valance band, and quasi-Fermi levels for electrons and holes, respectively.

reduced potential barrier height for holes and an increased potential barrier height for electrons compared to those of the reference device. The reduced potential barrier height for holes is attributed to the reduced positive effective E-field in the p-EBL layer as shown in Fig. 4(a) resulting in the enhanced hole injection efficiency from the p-EBL. The reduced positive effective E-field in the p-EBL layer could be originated from the higher relative static permittivity of the three-step graded $\mathrm{u}$-InGaN LQB layer. It is worth noting that the E-field in the p-EBL is inversely proportional to the relative static permittivity of the material, which is interfaced with the p-EBL. ${ }^{26-28}$ The improved efficiency of hole injection to the MQWs by incorporating the three-step graded u-InGaN LQB is confirmed by the simulated hole concentration distribution as shown in Fig. 5(a). The combining effect of the electron overflow reduction from MQWs and the hole injection enhancement by the application of the three-step graded $\mathrm{u}$-InGaN LQB, therefore, enhances the radiative recombination rates as shown in Fig. 5(b), which explains the enhancement of the optical output power and the EQE in

TABLE I. Energy barrier heights computed for the reference device and the proposed three-step graded u-InGaN LQB device at $20 \mathrm{~A} / \mathrm{cm}^{2}$.

\begin{tabular}{lccc}
\hline \hline Device & $\Delta \varnothing_{\mathrm{e}-\mathrm{LQB}}$ & $\Delta \varnothing_{\mathrm{e}-\mathrm{EBL}}$ & $\Delta \varnothing_{\mathrm{h}-\mathrm{EBL}}$ \\
\hline Reference device & $210 \mathrm{meV}$ & $325 \mathrm{meV}$ & $469 \mathrm{meV}$ \\
three-step graded u-InGaN LQB device & $362 \mathrm{meV}$ & $490 \mathrm{meV}$ & $358 \mathrm{meV}$ \\
\hline \hline
\end{tabular}
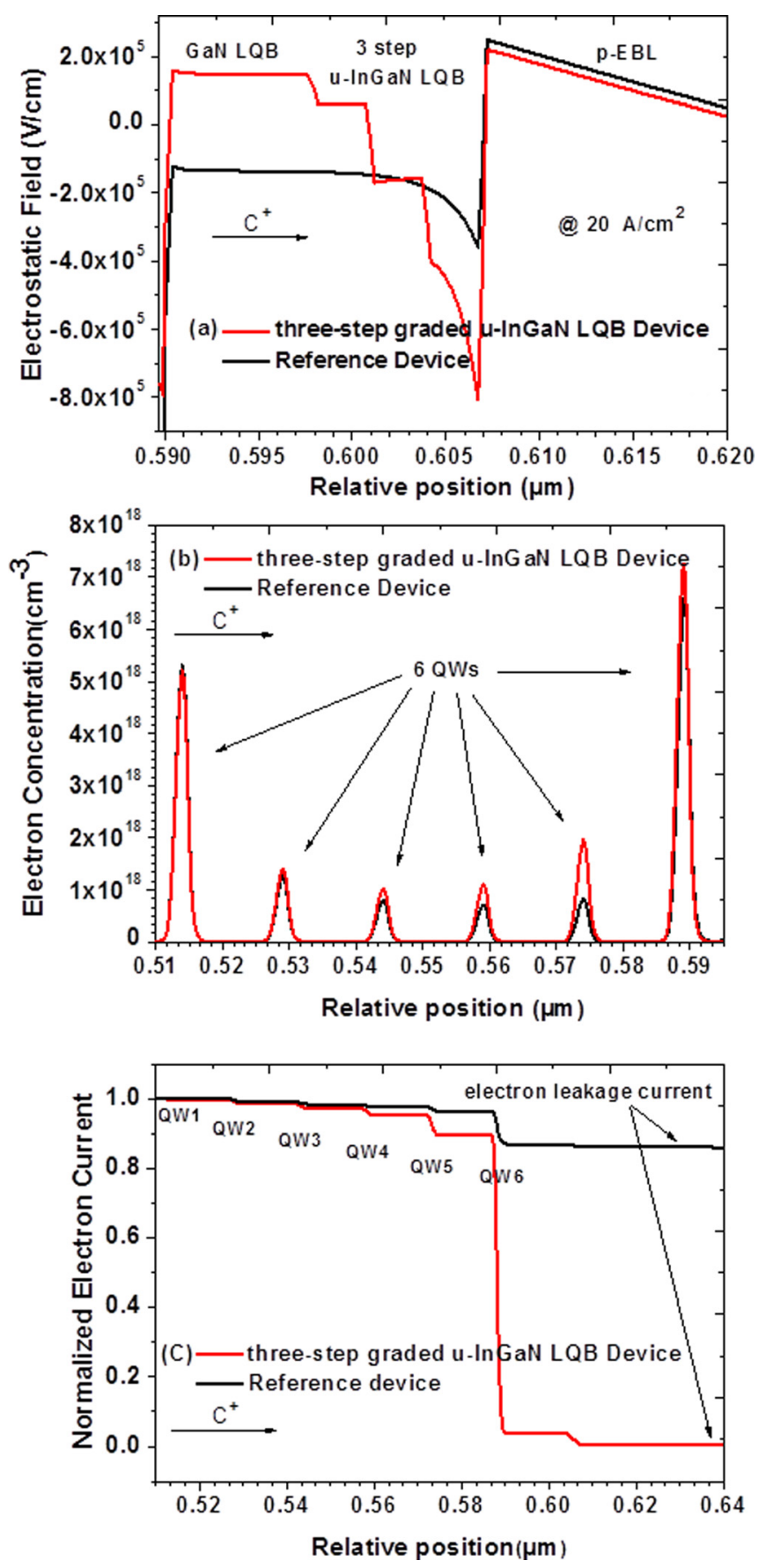

FIG. 4. (a) Electrostatic field, (b) electron concentration, and (c) normalized electron current simulated at $20 \mathrm{~A} / \mathrm{cm}^{2}$ across the InGaN/GaN QWs region in the reference device and the proposed three-step graded u-InGaN LQB device for comparison.

the three-step graded u-InGaN LQB device. Moreover, it is worth pointing out that negligibly small radiative recombination rate is observed in the three-step graded $\mathrm{u}$-InGaN layers due to insignificant smaller hole concentration and that the EL emission is predominantly contributed from the QWs, in particular largely in the 6th QW. From the experiment, as shown in Figs. 1(c) and 1(d)), a single EL emission with almost the same spectral width is observed, except the fact that a higher EL intensity level is obtained from the proposed device at different injection currents in Figs. 1(a) and 1(b), compared to the reference device.

In conclusion, the InGaN/GaN LED with the three-step graded $\mathrm{u}-\mathrm{InGaN}$ LQB inserted between the GaN LQB region 

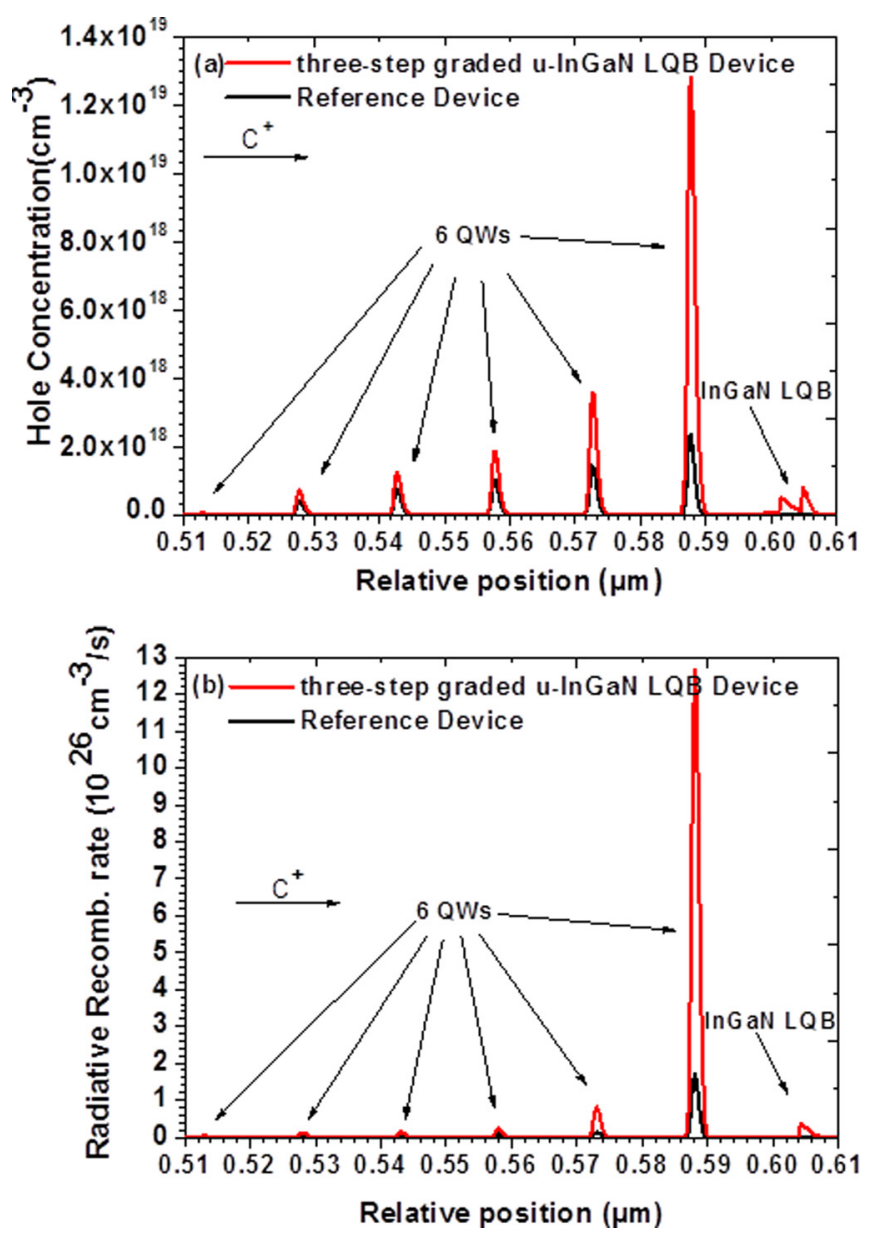

FIG. 5. The simulated (a) hole concentration and (b) radiative recombination rates at $20 \mathrm{~A} / \mathrm{cm}^{2}$ across the InGaN/GaN MQWs region for the reference device and the proposed three-step graded u-InGaN LQB device, respectively.

and the p-EBL has been proposed and studied in this work. Our findings indicate that the three-step graded $\mathrm{u}-\mathrm{InGaN}$ LQB can suppress the electron escape from the MQWs and improve the hole injection efficiency at the same time, and as a result, the optical power and the EQE are significantly improved as confirmed both experimentally and theoretically.

We gratefully acknowledge that this work was supported by the Singapore National Research Foundation under Grant Nos. NRF-CRP-6-2010-2 and NRF-RF-2009-09 and the Singapore Agency for Science, Technology and Research (A*STAR) SERC under Grant No. 112-120-2009.
${ }^{1}$ S. T. Tan, X. W. Sun, H. V. Demir, and S. P. DenBaars, IEEE Photon. J. 4, 613 (2012).

${ }^{2}$ S. Nagahama, N. Iwasa, M. Senoh, T. Matsushita, Y. Sugimoto, H. Kiyoku, T. Kozaki, M. Sano, H. Matsumura, H. Uemoto, K. Chocho, and T. Mukai, Jpn. J. Appl. Phys., Part 2 39, L647 (2000).

${ }^{3}$ T. Tojyo, T. Asano, M. Takeya, T. Hino, S. Kijima, S. Goto, S. Uchida, and M. Ikeda, Jpn. J. Appl. Phys., Part 1 40, 3206 (2001).

${ }^{4}$ M. H. Kim, M. F. Schubert, Q. Dai, J. K. Kim, E. F. Schubert, J. Piprek, and Y. Park, Appl. Phys. Lett. 91, 183507 (2007).

${ }^{5}$ M. F. Schubert, S. Chhajed, J. K. Kim, E. F. Schubert, D. D. Koleske, M. H. Crawford, S. R. Lee, A. J. Fischer, G. Thaler, and M. A. Banas, Appl. Phys. Lett. 91, 231114 (2007).

${ }^{6}$ M. F. Schubert, J. Xu, J. K. Kim, E. F. Schubert, M. H. Kim, S. Yoon, S. M. Lee, C. Sone, T. Sakong, and Y. Park, Appl. Phys. Lett. 93, 041102 (2008).

${ }^{7}$ J. Xie, X. Ni, Q. Fan, R. Shimada, U. Ozgur, and H. Morkoc, Appl. Phys. Lett. 93, 121107 (2008).

${ }^{8}$ I. V. Rozhansky and D. A. Zakheim, Phys. Status Solidi A 204, 227 (2007).

${ }^{9}$ I. A. Pope, P. M. Smowton, P. Blood, J. D. Thomson, M. J. Kappers, and C. J. Humphreys, Appl. Phys. Lett. 82, 2755 (2003).

${ }^{10}$ Y.-K. Kuo, J.-Y. Chang, and M.-C. Tsai, Opt. Lett. 35(19), 3285 (2010).

${ }^{11}$ C. S. Xia, Z. M. S. Li, W. Lu, Z. H. Zhang, Y. Sheng, W. D. Hu, and L. W. Cheng, J. Appl. Phys. 111, 094503 (2012).

${ }^{12}$ K. S. Kim, J. H. Kim, S. J. Jung, Y. J. Park, and S. N. Cho, Appl. Phys. Lett. 96, 091104 (2010).

${ }^{13}$ S.-H. Yen, M.-C. Tsai, M.-L. Tsai, S. Yu-Jiun, T.-C. Hsu, and Y.-K. Kuo, IEEE Photon. Technol. Lett. 21(14), 975 (2009).

${ }^{14}$ X. Ni, X. Li, J. Lee, S. Liu, V. Avrutin, Ü. Özgür, H. Morkoç, A. Matulionis, T. Paskova, G. Mulholland, and K. R. Evans, Appl. Phys. Lett. 97, 031110 (2010).

${ }^{15}$ R.-M. Lin, S.-F. Yu, S.-J. Chang, T.-H. Chiang, and S.-P. Chang, Appl. Phys. Lett. 101, 081120 (2012).

${ }^{16}$ Z. Liu, J. Ma, X. Yi, E. Guo, L. Wang, J. Wang, N. Lu, J. Li, I. Ferguson, and A. Melton, Appl. Phys. Lett. 101, 261106 (2012).

${ }^{17}$ T. Lu, S. Li, C. Liu, K. Zhang, and Y. Xu, Appl. Phys. Lett. 100, 141106 (2012).

${ }^{18}$ Y.-K. Kuo, Y.-H. Shih, M.-C. Tsai, and J.-Y. Chang, IEEE Photon. Technol. Lett. 23, 1630 (2011).

${ }^{19}$ J. Chen, G.-H. Fan, W. Pang, S.-W. Zheng, and Y.-Y. Zhang, IEEE Photon. Technol. Lett. 24, 2218 (2012).

${ }^{20}$ Y. K. Kuo, M. C. Tsai, S. H. Yen, T. C. Hsu, and Y. J. Shen, IEEE J. Quantum Electron. 46, 1214 (2010).

${ }^{21}$ S.-H. Yen, M.-L. Tsai, M.-C. Tsai, S.-J. Chang, and Y.-K. Kuo, IEEE Photon. Technol. Lett. 22, 1787 (2010).

${ }^{22}$ K. J. Vampola, M. Iza, S. Keller, S. P. DenBaars, and S. Nakamura, Appl. Phys. Lett. 94, 061116 (2009).

${ }^{23}$ M. F. Schubert and J. K. KIM, Int. J. High Speed Electron. Syst. 20, 247 (2011).

${ }^{24}$ Z.-H. Zhang, S. T. Tan, Z. Kyaw, Y. Ji, W. Liu, Z. Ju, N. Hasanov, X. W. Sun, and H. V. Demir, Appl. Phys. Lett. 102(19), 193508 (2013).

${ }^{25}$ V. Fiorentini, F. Bernardini, and O. Ambacher, Appl. Phys. Lett. 80, 1204 (2002).

${ }^{26}$ S.-H. Park and S.-L. Chuang, Appl. Phys. Lett. 76, 1981 (2000).

${ }^{27}$ A. D. Bykhovski, B. L. Gelmont, and M. S. Shur, J. Appl. Phys. 81, 6332 (1997).

${ }^{28}$ S. H. Park and S. L. Chuang, J. Appl. Phys. 87, 353 (2000). 